\title{
Extracting Family History of Patients From Clinical Narratives: Exploring an End-to-End Solution With Deep Learning Models
}

Xi Yang ${ }^{1,2}$, PhD; Hansi Zhang ${ }^{1}$, MSc; Xing $\mathrm{He}^{1}$, MSc; Jiang Bian ${ }^{1,2}, \mathrm{PhD}$; Yonghui $\mathrm{Wu}^{1,2}, \mathrm{PhD}$

${ }^{1}$ Department of Health Outcomes and Biomedical Informatics, College of Medicine, University of Florida, Gainesville, FL, United States

${ }^{2}$ Cancer Informatics Shared Resource, University of Florida Health Cancer Center, Gainesville, FL, United States

Corresponding Author:

Yonghui $\mathrm{Wu}, \mathrm{PhD}$

Department of Health Outcomes and Biomedical Informatics

College of Medicine

University of Florida

2004 Mowry Rd

Gainesville, FL, 32610

United States

Phone: 13522948436

Email: yonghui.wu@ufl.edu

\section{Abstract}

Background: Patients' family history (FH) is a critical risk factor associated with numerous diseases. However, FH information is not well captured in the structured database but often documented in clinical narratives. Natural language processing (NLP) is the key technology to extract patients' FH from clinical narratives. In 2019, the National NLP Clinical Challenge (n2c2) organized shared tasks to solicit NLP methods for FH information extraction.

Objective: This study presents our end-to-end FH extraction system developed during the $2019 \mathrm{n} 2 \mathrm{c} 2$ open shared task as well as the new transformer-based models that we developed after the challenge. We seek to develop a machine learning-based solution for FH information extraction without task-specific rules created by hand.

Methods: We developed deep learning-based systems for FH concept extraction and relation identification. We explored deep learning models including long short-term memory-conditional random fields and bidirectional encoder representations from transformers (BERT) as well as developed ensemble models using a majority voting strategy. To further optimize performance, we systematically compared 3 different strategies to use BERT output representations for relation identification.

Results: Our system was among the top-ranked systems (3 out of 21) in the challenge. Our best system achieved micro-averaged F1 scores of 0.7944 and 0.6544 for concept extraction and relation identification, respectively. After challenge, we further explored new transformer-based models and improved the performances of both subtasks to 0.8249 and 0.6775 , respectively. For relation identification, our system achieved a performance comparable to the best system $(0.6810)$ reported in the challenge.

Conclusions: This study demonstrated the feasibility of utilizing deep learning methods to extract FH information from clinical narratives.

(JMIR Med Inform 2020;8(12):e22982) doi: 10.2196/22982

\section{KEYWORDS}

family history; information extraction; natural language processing; deep learning

\section{Introduction}

Patients' family history $(\mathrm{FH})$ is a critical risk factor associated with numerous diseases [1-3] such as diabetes [4], coronary heart disease [5], and multiple types of cancers [6-9]. For example, a previous study showed that if a female patient has both her mother and sister having breast cancer, her relative risk [10] of having breast cancer increased 3.6 times compared with people without such FH [11]. Knowing the FH of patients can greatly help the prevention, diagnosis, and treatment of various diseases. However, FH is not well structured in current electronic health record databases but often documented as free text in clinical notes. Manually extracting patients' $\mathrm{FH}$ information is a labor-intensive and time-consuming procedure that cannot be scaled up. Natural language processing (NLP) is the key technology to build automated computational models 
to extract patients' FH from clinical narratives in their electronic health records.

In the past 2 decades, researchers have invested a significant amount of effort into developing various methods and tools to extract patients' information from clinical narratives [12-14]. The clinical NLP community has organized a series of shared tasks for retrieving various patients' information from clinical narratives including diseases or disorders [15-17], adverse drug events [18,19], and medical temporal relations [20]. Both rule-based and machine learning-based methods have been examined, and clinical NLP systems such as MetaMap [21], cTAKES [22], and CLAMP [23] have been developed. More recently, deep learning-based approaches have demonstrated superior performances in many NLP tasks [24]. For example, the long short-term memory-conditional random fields (LSTM-CRFs) architecture [25], which is a modified implementation of the recurrent neural network, has been widely adopted for named entity recognition (NER) tasks in both general and clinical domains. Later, a newly emerged bidirectional encoder representations from transformers (BERT) model achieved state-of-the-art performances in 20 NLP benchmarks in the general English domain [26] and demonstrated promising results in several clinical NLP tasks [27-29]. However, there are only a handful of studies focused on extracting $\mathrm{FH}$ of patients [30-32], which is more complicated than merely extracting information of the patients as it relates to various family members of the patient. FH often contains information from different aspects of the patients, including family members, their living status, and their diseases or disorders. Furthermore, patient's family members need to be characterized by family role (eg, mother) and family side (eg, maternal). Besides, there are limited clinical corpora annotated for $\mathrm{FH}$. The 2018 BioCreative/OHNLP Challenge $[33,34]$ is the first shared task focusing on FH extraction. During that challenge, Shi et al [35] explored a joint deep learning approach and achieved the best performance among all participated teams.
In 2019, the National NLP Clinical Challenge (n2c2) organized shared tasks to solicit advanced NLP methods for extracting FH information from clinical text. The 2019 n2c2 open shared task consisted of 2 subtasks: (1) NER for family members and observations (ie, diseases or disorders); and (2) identifying relations between family members, observations, and living status. Participants were required to identify mentions of $\mathrm{FH}$ and present a family member as a combination of family role (eg, mother) and family side (eg, maternal) and living status as a score derived from the healthy and alive state.

This paper presents our end-to-end FH extraction system developed during the $2019 \mathrm{n} 2 \mathrm{c} 2$ open shared task as well as new transformer models we developed after the challenge. During this challenge, we adopted an LSTM-CRF model for NER and a BERT-based model for relation identification. Our best submission was ranked fifth in subtask 1 and third in subtask 2. After the challenge, we further explored a BERT-based model for NER and demonstrated better performances in both subtasks.

\section{Methods}

\section{Data}

This study used the data set developed by the 2019 n2c 2 open shared task organizers consisting of 216 clinical notes extracted from the Mayo Clinic data warehouse. The organizers split the corpus into a training set of 99 notes and a test set of 117 notes. Three types of concepts were annotated, including family members, observations (ie, diseases and disorders), and living status. There are also 2 types of relations annotated among family members, observations, and living status. The organizers provided annotations at (1) entity level (ie, the words and phrases about $\mathrm{FH}$ ), and (2) document level, where the multiple mentions of the same FH were aggregated. Table 1 shows the descriptive statistics of the corpus.

Table 1. Descriptive statistics of the challenge data set.

\begin{tabular}{|c|c|c|}
\hline \multirow[t]{2}{*}{ Corpus information, annotation type, and annotation category } & \multicolumn{2}{|c|}{2019 n2c2 family history challenge corpus } \\
\hline & Training set & Test set \\
\hline Number of notes & 99 & 117 \\
\hline \multicolumn{3}{|l|}{ Entity-level annotation } \\
\hline \multicolumn{3}{|l|}{ Concept } \\
\hline Family members & 803 & N/A \\
\hline Observations & 978 & N/A \\
\hline Living status & 415 & N/A \\
\hline \multicolumn{3}{|l|}{ Document-level annotation } \\
\hline \multicolumn{3}{|l|}{ Concept } \\
\hline Family members & 667 & 638 \\
\hline Observations & 930 & 983 \\
\hline \multicolumn{3}{|l|}{ Relation } \\
\hline Family members—observations & 740 & 755 \\
\hline Family members—living status & 376 & 349 \\
\hline
\end{tabular}




\section{The Family History Extraction System}

Figure 1 shows the system architecture for our end-to-end FH extraction system. Our system has 5 modules including preprocessing, NER, classification, relation identification, and postprocessing. The preprocessing module contains standard NLP procedures including tokenization, sentence boundary detection, and data format transformation. In the NER module, we explored state-of-the-art NLP models, including LSTM-CRFs and BERT to identify FH concepts. The relation identification module applied deep learning models to determine the relations among FH concepts. The postprocessing module aggregated the entity-level results to the document level for both concept extraction and relation identification subtasks.

Figure 1. Overview of our family history extraction system.

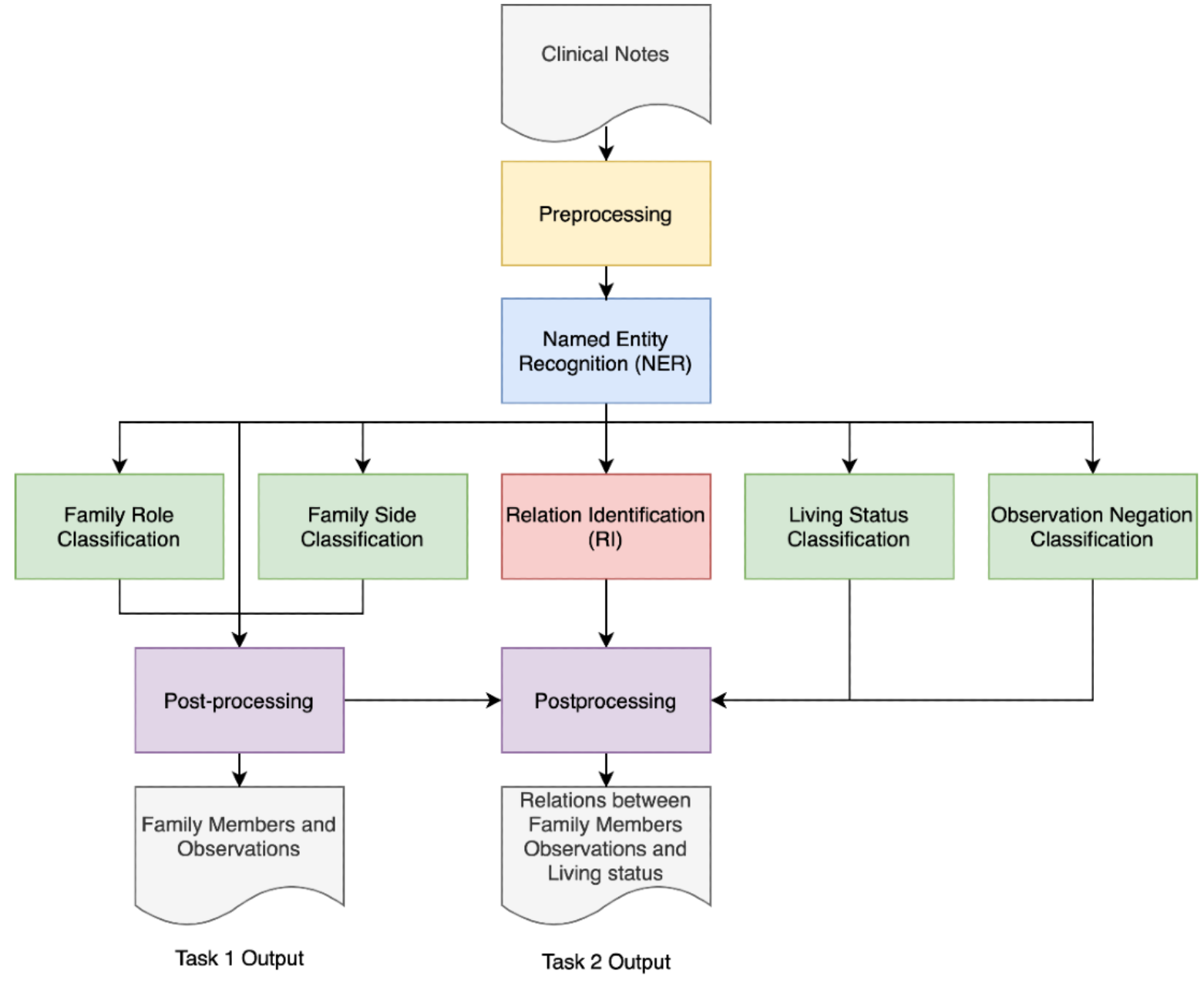

\section{Extracting Family History Concepts}

The concept extraction subtask focused on detecting the mentions of family members and observations. We approached this subtask as a typical NER problem and applied deep learning-based models. Following the standard machine learning-based NER procedure, we converted the annotations using the beginning-inside-outside (BIO) tagging scheme $[36,37]$, where "B" indicates the first token of a concept, "I" indicates tokens inside of a concept, and " $\mathrm{O}$ " indicates tokens that do not belong to any concepts. Thus, we converted information extraction problem into a sequence labeling task to assign each word with one of the predefined NER labels ("B," "I," or "O"). We explored 2 deep learning-based models including LSTM-CRFs and BERT.

Previous studies [38-41] have shown that adopting an ensemble method could further improve the clinical NER performances.
Thus, we adopted the majority voting strategy to integrate the different NER models as shown in Figure 2. More specifically, we randomly (based on a random seed) split the training data into a short training data and a validation data at a 9:1 ratio. We trained deep learning models using the short training data and selected the best checkpoints based on the model performance on the validation data. By repeating the procedure 5 times with different random seeds, we obtained 5 different models. In each training procedure, we used different short training data and validate data but the same hyperparameters (ie, the optimized hyperparameters used for training the single BERT NER model). Then, the majority voting strategy was used to vote among the 5 models. Here, we use a suffix "-EN" to indicate the ensemble method. For example, we used "LSTM-CRFs-EN" to denote the ensemble model of LSTM-CRFs, and "BERT-EN" to denote the ensemble model of using BERT. 
Figure 2. The majority voting strategy to ensemble NER models. BERT: bidirectional encoder representations from transformers; NER: named entity recognition.

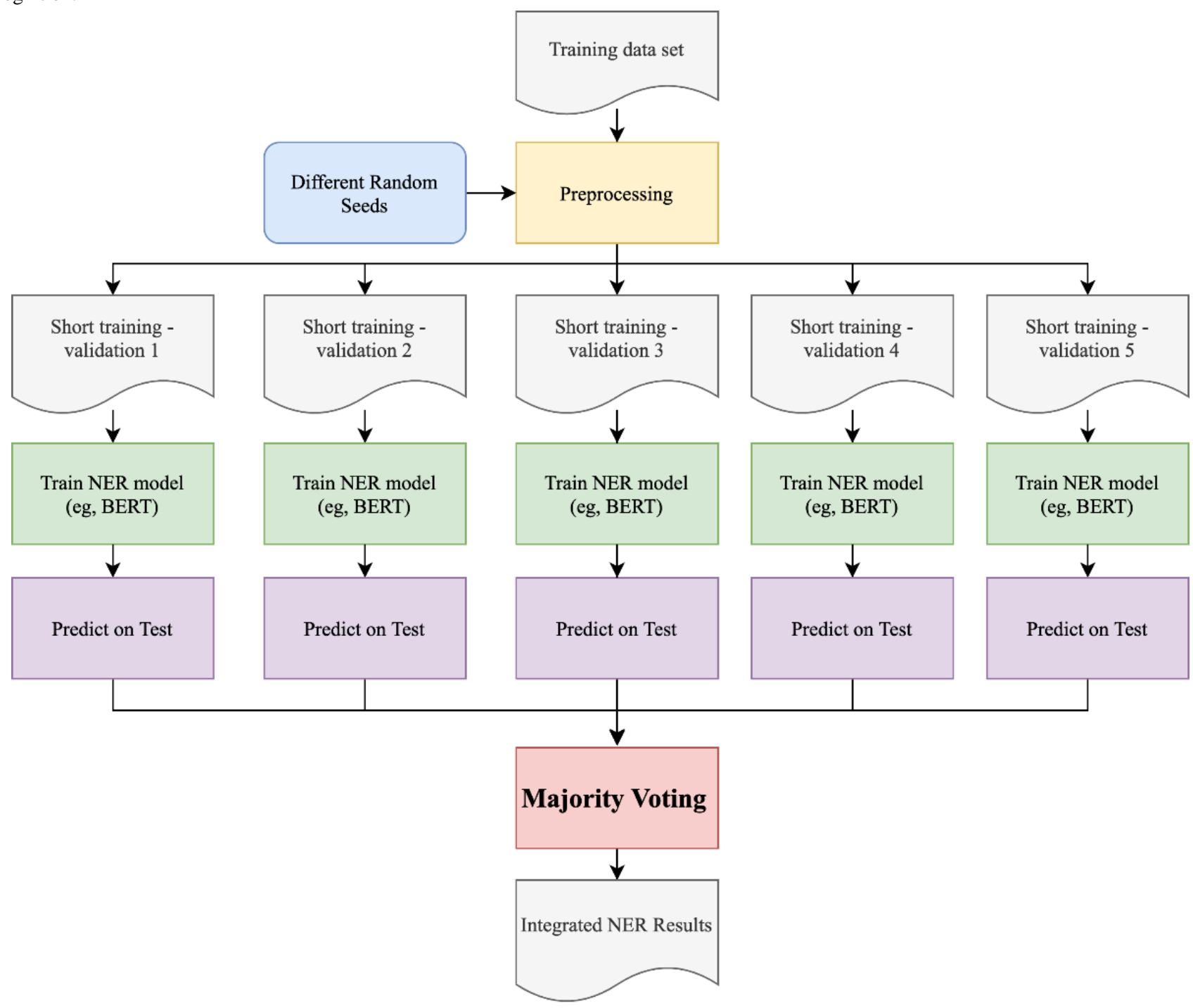

\section{Determining Family Role and Family Side}

This task is to determine the family role and family side for the mentions of FH. There are a total number of 15 types of family roles defined in this challenge, including father, mother, sister, parent, brother, grandmother, grandfather, grandparent, daughter, son, child, cousin, sibling, aunt, and uncle. There are 3 predefined family sides including maternal, paternal, and not applicable. We approached the 2 tasks as classification problems. Previous studies $[35,42]$ approached the 2 tasks using rule-based methods; here, we applied deep learning-based classification methods as machine learning-based methods have shown a better generalizability.

\section{Relation Identification}

Typically, relation identification consists of 2 steps: (1) determine whether there is a relation between 2 entities; and (2) classify the correct relation type. In this study, we formulated the relation identification as a binary classification problem. We presented each relation as a pair of 2 entities and used contextual information around the entities to classify these pairs into categories as "in-relation" or "nonrelation" (no relation between entities). Then, we further categorized the "in-relation" entity pairs into either "family member-living status" group or "family member-observation" group based on the entity types: if 1 of the entities in an entity pair is observation, we classify it as "family member-observation"; if one of the entities in an entity pair is living status, we classify it as "family member-living status."

\section{Candidate Concept Pairs Generation}

Theoretically, there might be relations between any pair of $\mathrm{FH}$ concepts. Thus, a naïve way is to generate candidate pairs from all combinations of clinical concepts in document level. However, a previous study [43] has reported that this method often generates too many negative samples (ie, nonrelation), causing an extremely imbalanced positive-to-negative sample ratio. To alleviate this issue, we applied the following heuristic rule to reduce the combinations: only keep the concept pairs composed of a family member entity as the first element and a nonfamily member entity as the second element. We also looked into the cross-distance of pairs-defined as the number of sentence boundaries between the 2 entities (eg, 0 for single-sentence relations, and 1 for relations across 2 sentences). In the training set, the cross-distance ranges from 0 to 10 and we found that $96 \%$ of the annotated relations have 
cross-distances less than 3. Therefore, we only consider candidate pairs with cross-distances less than 3 . Previous studies $[44,45]$ developed individual classifiers to handle relations with different cross-distance; here, we developed a unified BERT-based classifier to handle all candidate pairs with various cross-distances as the BERT model is able to learn both tokenand sentence-level representations.

\section{Handling Negations}

In this study, we approached negation detection as a binary classification problem-classify the observation entity into 2 predefined categories including "negated" and "non-negated." We developed a BERT-based classifier for negation detection. In our system, we performed the negation detection for each observation entity and then integrated the results into relations. We only used the negation annotations from the challenge data set and did not use any external resources.

\section{Assessing the Living Status Scores}

For the relations between "family member-living status," the participants were required to assess the living status using scores of 0,2 , or 4 , where 0 indicates not alive, 2 indicates alive but not healthy, and 4 indicates alive and healthy. We approached this task as a classification task - to categorize a living status entity into one of 3 score categories (ie, 0, 2, and 4). We developed a BERT-based classifier to classify each living status entity into a category according to its context.

\section{Deep Learning Models}

\section{LSTM-CRFS}

In this study, we adopted an LSTM-CRFs architecture proposed by Lample et al [25]. The model has 2 bidirectional LSTM layers: one for learning representations at the character level and the other for learning those at the word level. The model utilizes a CRFs layer to decode the LSTM hidden states to BIO tags. We screened 4 different word embeddings following a similar procedure reported in our previous study [46] and found that the Common Crawl embeddings-released by Facebook and trained using the fastText on the Common Crawl data set [47]-achieved better performance compared to other embeddings on a validation data set. Thus, we used the Common Crawl embeddings for all LSTM-CRFs models.

\section{BERT}

The BERT model is a multilayer transformer encoder model implemented using the self-attention mechanism [48], which is pretrained by combining the masked language modeling method and the next sentence prediction task. BERT has 2 versions featuring different model sizes, including a BASE version with 12 transformer layers and 110 million parameters, and a LARGE version with 24 transformer layers and 340 million parameters [26]. There are 2 steps to apply BERT for various downstream NLP, including (1) pretraining a BERT model using large unlabeled corpora and (2) fine-tuning the pretrained model using task-specific annotated corpora. In this study, we adopted the general pretrained BERT-LARGE model and fine-tuned it individually for each subtask (ie, concept extraction and relation identification) using the annotated data set developed in this challenge. We denoted the BERT-based NER model as BERT-ner, and the BERT-based family member attributes (ie, family role, side of family, negation, living status) classification module as BERT-cls and relation extraction module as BERT-rel.

Figure 3 illustrates the fine-tuning procedure for BERT. For token $\mathrm{Tok}_{\mathrm{i}}$, its input embedding and contextual representation are denoted as $\mathrm{Emb}_{\mathrm{i}}$ and $\mathrm{T}_{\mathrm{i}}$. The [CLS] and [SEP] are 2 special symbols designed to format the input sequences. In this study, we also introduced a pair of entity marker including [S] and [E] to differentiate the target entity from other entities in the same sentence, where $[\mathrm{S}]$ indicates the start position and $[\mathrm{E}]$ indicates the end position. For NER (Figure 3A), the input for BERT model is a sequence of tokens, and the output is a sequence of distributed representation. Then, we used a linear layer to calculate a score for each BIO tag. Based on the entities, we developed classifiers to determine related attributes (Figure 3B). To distinguish between the target entity and other entities in the same sentence, we inserted entity markers (ie, $[\mathrm{S}]$ and $[\mathrm{E}]$ ) in front of and after the target entity. For example, the input sequenced in Figure 3B contains the target entity (ie, Tok 1 and $\mathrm{Tok}_{2}$ ) surrounded by the entity markers and other entities (eg, $\mathrm{Tok}_{\mathrm{n}}$ ). Then, we concatenated the representations corresponding to the [CLS] and [S] tokens and calculated a score for each predefined class label using a linear layer. For relation identification (Figure 3C), we determined the relation type based on the contextual information of 2 concepts in a relation. Therefore, the input consisted of 2 sentences linked by the special token [SEP], where each sentence contains 1 of the 2 entities in the relation. We used 2 sets of entity markers (ie, [S1], [E1], and [S2], [E2]) to label the entities. If the 2 entities of a relation are in the same sentence, then the 2 model-input sentences are the same but with different entity markers. To determine the relation category, we concatenated the representations from [CLS] and 2 start position entity makers ([S1] and [S2]) and used a linear layer to calculate a score for each predefined relation type. 
Figure 3. Illustration of BERT models for (A) NER, (B) family member attributes (including side and role of family members, negation of observations, and living scores) classification, and (C) relation extraction. BERT: bidirectional encoder representations from transformers; NER: named entity recognition.

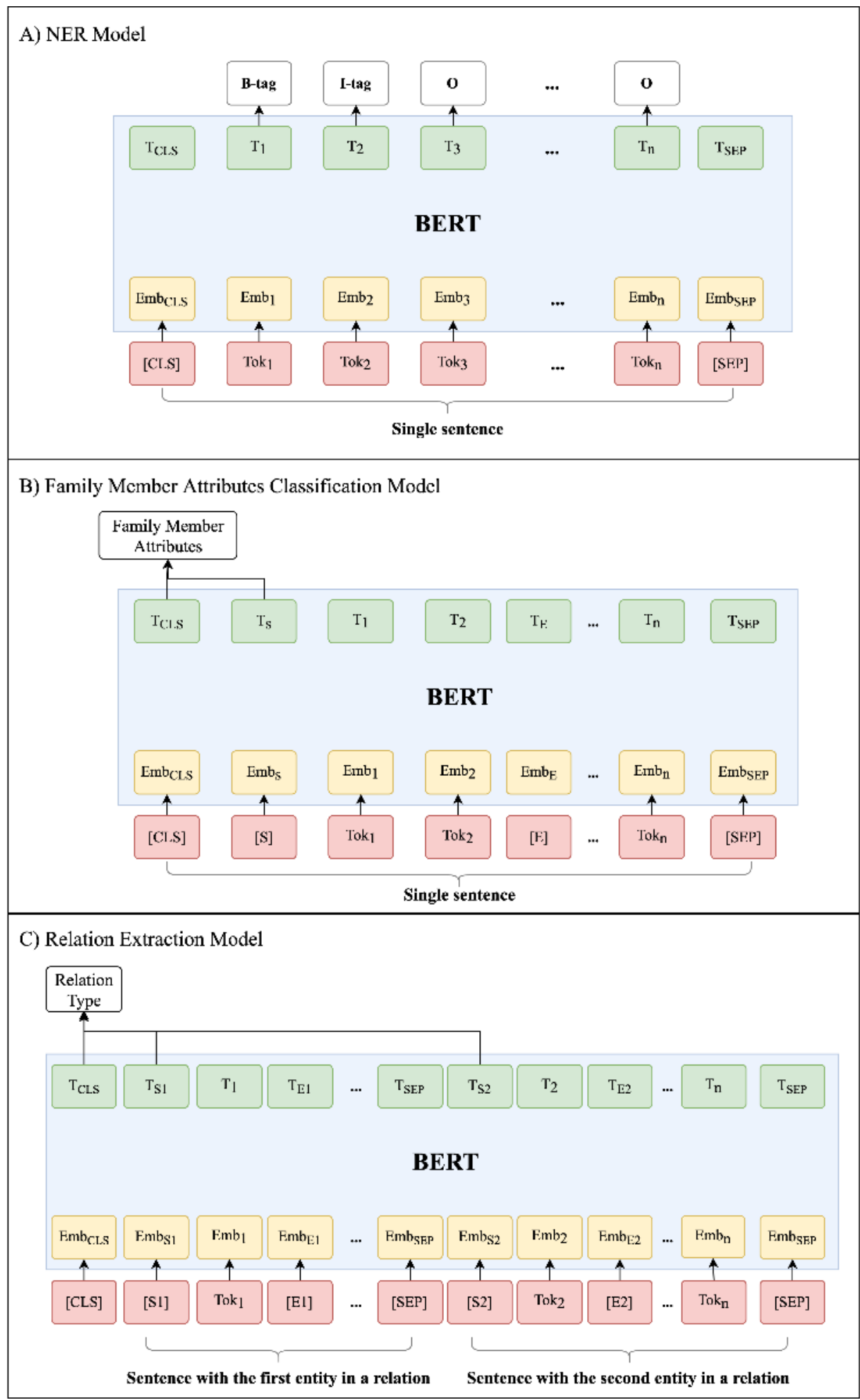

\section{Experiments and Evaluations}

In this study, we reused the LSTM-CRFs model developed in our previous study [49] and implemented the BERT-based models on top of the Transformers library [50] implemented in PyTorch [51]. We used the following parameters to initialize the LSTM-CRFs: the character embedding dimension was 25 , the word embedding dimension was 100 , the character-level bidirectional LSTM layer dimension was 25, the word-level bidirectional LSTM layer was 100 with a dropout probability of 0.5 , the learning rate was fixed at 0.005 , and the stochastic gradient descending applied a gradient clapping at $[-5.0,5.0]$. The character embeddings were randomly initialized and the word embeddings were initiated using embeddings from fastText [47] (ie, containing 2 million word vectors trained on Common 
Crawl). We initialized all BERT-based models using the BERT-LARGE pretrained on the general English corpus and fine-tuned them with the default model parameter settings. To train NER models, we randomly (using random seeds for reproducibility) split the original training set (99 notes) into a short training set of 89 notes and a development set of 10 notes. The best NER models were selected according to the performance on the development set. We optimized 2 hyperparameters, including the number of epochs and batch size, via fivefold cross-validation. Table 2 summarizes the optimized hyperparameters. We conducted all experiments using 2 NVIDIA P6000 graphics processing units (GPUs). We used the official evaluation script provided by the 2019 n2c2 open shared task organizers to calculate the evaluation scores on the test set. Evaluation metrics as micro-averaged precision, recall, and F1 score were used for both subtask 1 and subtask 2.

Table 2. The optimized hyperparameters of BERT-based models for various tasks.

\begin{tabular}{lllll}
\hline Task & Pretrained model & Number of epochs & Batch size & Learning rate \\
\hline NER $^{\mathrm{a}}$ & BERT -LARGE & 30 & 4 & 8 \\
Negation classification & BERT-LARGE & 5 & 4 & $1.00 \times 10^{-05}$ \\
Side of family classification & BERT-LARGE & 10 & 8 & $1.00 \times 10^{-05}$ \\
Role of family classification & BERT-LARGE & 5 & 8 & $1.00 \times 10^{-05}$ \\
Living status classification & BERT-LARGE & 6 & 16 & $1.00 \times 10^{-05}$ \\
Relation identification & BERT-LARGE & 12 & $2.00 \times 10^{-05}$ \\
\hline
\end{tabular}

${ }^{\mathrm{a}} \mathrm{NER}$ : named entity recognition.

${ }^{\mathrm{b}}$ BERT: bidirectional encoder representations from transformers.

\section{Results}

Table 3 compares our 4 systems for conception extraction and relation identification. Our best submission during the original challenge (LSTM-CRFs-EN + BERT- $c l s$ +BERT-rel) achieved F1 scores of 0.7944 and 0.6544 for subtask 1 and subtask 2, respectively, which is the third best system of this challenge among 17 participants. After the challenge, we further explored the BERT model for NER and the combination of
BERT-ner-EN, BERT-cls, and BERT-rel achieved better F1 scores of 0.8249 and 0.6775 for the 2 subtasks, respectively. Compared to our best system developed during the challenge (LSTM-CRFs-EN + BERT-cls + BERT-rel), the new system (BERT-ner-EN + BERT-cls + BERT-rel) improved the F1 scores by 0.0305 and 0.0235 for the 2 subtasks, respectively. Our best relation identification performance was comparable to the best result reported in this challenge $(0.6775$ from us versus 0.6810 reported in this challenge).

Table 3. The micro-average performances for concept extraction and relation identification.a

\begin{tabular}{|c|c|c|c|c|c|c|}
\hline \multirow[t]{2}{*}{ Models } & \multicolumn{3}{|c|}{ Subtask 1 (concept extraction) } & \multicolumn{3}{|c|}{ Subtask 2 (relation identification) } \\
\hline & Precision & Recall & F1 score & Precision & Recall & F1 score \\
\hline $\mathrm{LSTM}^{\mathrm{a}}-\mathrm{CRFs}^{\mathrm{b}}+\mathrm{BERT}^{\mathrm{c}}-c l s+\mathrm{BERT}-r e l$ & 0.7760 & 0.8087 & 0.7920 & 0.7343 & 0.5465 & 0.6266 \\
\hline LSTM-CRFs-EN + BERT- $c l s+$ BERT-rel ${ }^{\mathrm{d}}$ & 0.7969 & 0.7920 & 0.7944 & 0.6995 & 0.6184 & 0.6544 \\
\hline BERT-ner + BERT-cls + BERT-rel & 0.8060 & 0.8105 & 0.8083 & 0.7140 & $0.6252^{\mathrm{e}}$ & 0.6667 \\
\hline BERT-ner-EN + BERT-cls + BERT-rel & $0.8301^{\mathrm{e}}$ & $0.8198^{\mathrm{e}}$ & $0.8249^{\mathrm{e}}$ & $0.7421^{\mathrm{e}}$ & 0.6233 & $0.6775^{\mathrm{e}}$ \\
\hline
\end{tabular}

${ }^{\mathrm{a}}$ LSTM: long short-term memory.

${ }^{\mathrm{b}} \mathrm{CRF}$ : conditional random fields.

${ }^{c}$ BERT: bidirectional encoder representations from transformers.

${ }^{\mathrm{d}}$ Our best system developed during the challenge.

${ }^{\mathrm{e}}$ The best performances.

Table 4 compares the detailed performance of LSTM-CRFs and BERT-ner for FH extraction. Compared with LSTM-CRFs, the BERT-ner model achieved a remarkably higher F1 score for the observation concepts ( 0.8094 for BERT-ner versus 0.7833 for LSTM-CRFs), but marginally lower performance for the family member concepts (0.8066 for BERT-ner versus 0.8069 for LSTM-CRFs). Table 4 also demonstrated that our ensemble strategy improved the performance of FH extraction. For example, the BERT-ner-EN, which was ensembled from 5 different BERT-ner models, outperformed the single BERT-ner model by about $2 \%$ for family members and about $1.5 \%$ for observations. 
Table 4. A comparison of LSTM-CRFs and BERT for subtask 1 (concept extraction).

\begin{tabular}{|c|c|c|c|}
\hline Model and concept & Precision & Recall & F1 score \\
\hline \multicolumn{4}{|l|}{ LSTM-CRFs $^{\mathrm{a}, \mathrm{b}}$} \\
\hline Family member & 0.8480 & 0.7686 & 0.8069 \\
\hline Observation & 0.7382 & 0.8342 & 0.7833 \\
\hline \multicolumn{4}{|l|}{ LSTM-CRFs-EN } \\
\hline Family member & 0.8451 & 0.7868 & 0.8149 \\
\hline Observation & 0.7685 & 0.7953 & 0.7817 \\
\hline \multicolumn{4}{|l|}{ BERT $^{\mathrm{c}}-n e r$} \\
\hline Family member & 0.8059 & 0.8072 & 0.8066 \\
\hline Observation & 0.8061 & 0.8127 & 0.8094 \\
\hline \multicolumn{4}{|l|}{ BERT-ner-EN } \\
\hline Family member & 0.8294 & 0.8229 & 0.8261 \\
\hline Observation & 0.8306 & 0.8178 & 0.8241 \\
\hline
\end{tabular}

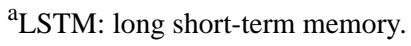

${ }^{\mathrm{b}} \mathrm{CRFs}$ : conditional random fields.

${ }^{c}$ BERT: bidirectional encoder representations from transformers.

Table 5 compares the performance of relation identification for member-living status" and "family member-observation" each relation category. Similar to the concept extraction results, relations, respectively. Compared to the LSTM-CRFs, the the BERT-ner-EN + BERT-cls + BERT-rel system achieved

BERT-ner-based systems achieved better recalls. the best F1 scores of 0.6821 and 0.6760 for the "family

Table 5. The category-level performances for subtask 2 (relation identification).

\begin{tabular}{|c|c|c|c|}
\hline Model and relation & Precision & Recall & $\mathrm{F} 1$ \\
\hline \multicolumn{4}{|c|}{ LSTM-CRFs $^{\mathrm{a}, \mathrm{b}}+$ BERT $^{\mathrm{c}}$-cls + BERT-rel } \\
\hline Family member-living status & 0.7039 & 0.6132 & 0.6554 \\
\hline Family member-observation & 0.7452 & 0.5269 & 0.6174 \\
\hline \multicolumn{4}{|c|}{ LSTM-CRFs-EN + BERT-cls + BERT-rel } \\
\hline Family member-living status & 0.6773 & 0.6676 & 0.6724 \\
\hline Family member-observation & 0.7071 & 0.5993 & 0.6487 \\
\hline \multicolumn{4}{|l|}{ BERT-ner + BERT-cls + BERT-rel } \\
\hline Family member-living status & 0.6583 & 0.6734 & 0.6657 \\
\hline Family member-observation & 0.7341 & 0.6111 & 0.6670 \\
\hline \multicolumn{4}{|c|}{ BERT-ner-EN + BERT-cls + BERT-rel } \\
\hline Family member-living status & 0.6912 & 0.6734 & 0.6821 \\
\hline Family member-observation & 0.7603 & 0.6086 & 0.6760 \\
\hline
\end{tabular}

${ }^{\text {a}}$ LSTM: long short-term memory.

${ }^{\mathrm{b}} \mathrm{CRFs}$ : conditional random fields.

${ }^{c}$ BERT: bidirectional encoder representations from transformers.

\section{Discussion}

\section{Overview}

Patients' FH is a critical risk factor associated with numerous diseases. Clinical NLP systems that automatically extract FH from clinical narrative are needed for many clinical studies and applications. The $2019 \mathrm{n} 2 \mathrm{c} 2$ organized shared tasks to assess current NLP methods for FH information extraction from clinical narratives. We participated in both subtasks and our system (LSTM-CRFs-EN + BERT-cls + BERT-rel) achieved the third best performance (F1 of 0.6544) among all the 21 submitted systems from 17 teams that participated in subtask 2. After the challenge, we further explored the BERT models 
for the concept extraction and improved our system in both concept extraction and relation identification.

\section{Principal Findings}

We observed that the BERT-ner model achieved both better precision $(0.8060$ versus 0.7760$)$ and recall $(0.8105$ versus 0.8087) for clinical concept extraction compared to the LSTM-CRFs, which is consistent with a recent study by $\mathrm{Si}$ et al [52]. We also noticed that the single BERT-ner mode even achieved a higher F1 score of 0.8083 than the ensembled LSTM-CRFs model (LSTM-CRFs-EN with F1 score of 0.7944). Ensemble is an effective strategy to further improve the performance of NER. For example, the ensembled BERT model (ie, BERT-ner-EN, which was ensembled from 5 individual BERT-ner models) improved the concept extraction performance to 0.8249 , compared to the single BERT model (F1 score of 0.8083). The performance improvement of the ensembled model was mainly in precision, suggesting that the ensembled models may reduce the classification errors in NER. However, further studies should examine whether our observation is related to the size of training corpus (relatively small, only 99 notes).

Most of the previous studies applied rule-based solutions to determine the family roles and family sides [34]. In this study, we adopted a pure machine learning-based solution. The experimental results showed that the BERT-based classifiers were feasible to determine the family roles, family sides, negation of observations, and living status scores. Another advantage of our method is that machine learning-based models generally have a better generalizability than rule-based systems and are easy to scale up. FH information has many variations from one patient to another, which makes the development of rules time-consuming and expensive.

In our system, we only used the sentences containing the concepts to classify the family member attributes. We also examined a strategy to include both the proceeding and following sentences. However, the experimental results based on the fivefold cross-validation on the training set showed that adding the context information did not improve the performance. One potential reason may be that most of the key information for classifying the family member attributes is located in the same sentence where the concepts (ie, family member or observation) are located. Besides, there might be potential noises brought in when including the context sentences.

A previous study [53] examined various input encoding and output representation of using BERT for relation extraction, and concluded that using representations aggregated from the start position entity markers (eg, [S1] and [S2] in Figure 3C) was the best practice. In this study, we re-evaluated 3 types of BERT output representations, including (1) the representation of the [CLS] only, (2) the representations aggregated from the start position entity markers, and (3) the representations aggregated from the [CLS] and the start position entity markers. Our results showed that option (3) led to a remarkably higher averaged F1 score (0.8975) compared to the other 2 representations $(0.8851$ and 0.8904$)$. A possible reason is that the representations captured in the special token [CLS] and the representations of the start position markers contain contextual information that is complement to each other. Further studies are needed to continue examining more efficient methods for encodings and representations.

This study has limitations. First, there are limited clinical corpora for FH-related information extraction as annotating clinical notes is expensive and time-consuming. A potential solution is to use data augmentation techniques such as generative adversarial networks, which have been applied for medical imaging data $[54,55]$. There are preliminary research works demonstrating that generative adversarial networks could be utilized to synthesize clinical text [56]. Second, our system is a 2-stage pipeline where the errors generated in the NER will be propagated to relation extraction. We will explore potential solutions such as joint learning algorithms to alleviate this issue in our future work.

\section{Error Analysis}

Table 6 shows the confusion matrix generated for the concept extraction (subtask 1) based on our best NER model (ie, BERT-ner-EN). The confusion matrix showed that our system could efficiently identify family member entities. However, it is challenging for our system to differentiate the nonconcept terms for both family members and observations. For concept extraction, our system had relatively lower performances for "parent," "grandparent," "child," and "siblings." One possible reason is that the training set contains limited annotations of these entities. For example, the "parent" entity only appeared once and the "grandparent" entities appeared 6 times in the training data set. We also found that our system identified some observations not annotated in the test set. For example, in the sentence "The father also had a history of vascular surgery, a long history of smoking, and has had hip replacement," our system extracted observations of "vascular surgery," "smoking," and "hip replacement," which were annotated in the challenge corpus. 
Table 6. The confusion matrix table for the NER (subtask 1). ${ }^{\mathrm{a}}$

\begin{tabular}{llll}
\hline Entity type & Model prediction & & $\mathrm{NC}^{\mathrm{d}}$ \\
\hline FM & $\mathrm{FM}^{\mathrm{b}}$ & $\mathrm{OB}^{\mathrm{c}}$ & 113 \\
$\mathrm{OB}$ & 525 & 0 & 178 \\
$\mathrm{NC}$ & 0 & 799 & $\mathrm{~N} / \mathrm{A}^{\mathrm{e}}$ \\
\hline
\end{tabular}

${ }^{\mathrm{a}} \mathrm{FM}, \mathrm{OB}$, and $\mathrm{NC}$ are considered gold standard.

${ }^{b}$ FM: family members.

${ }^{\mathrm{c}} \mathrm{OB}$ : observations.

${ }^{\mathrm{d}} \mathrm{NC}$ : not a concept.

${ }^{\mathrm{e}} \mathrm{N} / \mathrm{A}$ : not applicable.

\section{Conclusions}

Extracting patients' FH information from clinical narratives is a challenging NLP task. This study demonstrated the efficiency of deep learning-based NLP models for extraction of FH. Our system and pretrained models can be accessed at [57]. We believe our system could help other researchers to extract and leverage patient's FH documented in clinical narratives in their studies.

\section{Acknowledgments}

Research reported in this publication was supported by (1) the University of Florida Clinical and Translational Science Institute, which is supported in part by the NIH National Center for Advancing Translational Sciences under award number UL1TR001427; (2) the Patient-Centered Outcomes Research Institute (PCORI) under award number ME-2018C3-14754; (3) the Centers for Disease Control and Prevention (CDC) under award number U18DP006512; (4) the NIH National Cancer Institute (NCI) under award number R01CA246418; (5) the NIH National Institute on Aging under award number R21AG061431-02S1. The content is solely the responsibility of the authors and does not necessarily represent the official views of the National Institutes of Health and Patient-Centered Outcomes Research Institute. We thank the n $2 \mathrm{c} 2$ organizers for providing the annotated corpus and the guidance for this challenge. We gratefully acknowledge the support of NVIDIA Corporation for the donation of the GPUs used for this research.

\section{Authors' Contributions}

$\mathrm{XY}$, JB, and YW were responsible for the overall design, development, and evaluation of this study. HZ and XH were involved in conducting experiments and result analysis. XY, JB, and YW did the writing and editing of this manuscript. All authors reviewed the manuscript critically for scientific content, and all authors gave final approval of the manuscript for publication.

\section{Conflicts of Interest}

None declared.

\section{References}

1. Scheuner MT, Wang S, Raffel LJ, Larabell SK, Rotter JI. Family history: a comprehensive genetic risk assessment method for the chronic conditions of adulthood. Am J Med Genet 1997 Aug 22;71(3):315-324. [doi: 10.1002/(sici)1096-8628(19970822)71:3<315::aid-ajmg12>3.0.co;2-n] [Medline: 9268102 ]

2. Rich E, Burke W, Heaton C, Haga S, Pinsky L, Short M, et al. Reconsidering the family history in primary care. J Gen Intern Med 2004 Mar;19(3):273-280 [FREE Full text] [doi: 10.1111/j.1525-1497.2004.30401.x] [Medline: 15009784]

3. Guttmacher AE, Collins FS, Carmona RH. The family history--more important than ever. N Engl J Med 2004 Nov 25;351(22):2333-2336. [doi: 10.1056/NEJMsb042979] [Medline: 15564550]

4. Harrison TA, Hindorff LA, Kim H, Wines RC, Bowen DJ, McGrath BB, et al. Family history of diabetes as a potential public health tool. Am J Prev Med 2003 Feb;24(2):152-159. [doi: 10.1016/s0749-3797(02)00588-3] [Medline: 12568821]

5. Williams RR, Hunt SC, Heiss G, Province MA, Bensen JT, Higgins M, et al. Usefulness of cardiovascular family history data for population-based preventive medicine and medical research (the Health Family Tree Study and the NHLBI Family Heart Study). Am J Cardiol 2001 Jan 15;87(2):129-135. [doi: 10.1016/s0002-9149(00)01303-5] [Medline: 11152826]

6. Ramsey SD, Yoon P, Moonesinghe R, Khoury MJ. Population-based study of the prevalence of family history of cancer: implications for cancer screening and prevention. Genet Med 2006 Oct;8(9):571-575 [FREE Full text] [doi: 10.1097/01.gim.0000237867.34011.12] [Medline: 16980813 ]

7. Pharoah PD, Ponder BA. The genetics of ovarian cancer. Best Pract Res Clin Obstet Gynaecol 2002 Aug;16(4):449-468. [doi: 10.1053/beog.2002.0296] [Medline: 12413928] 
8. Johns LE, Houlston RS. A systematic review and meta-analysis of familial colorectal cancer risk. Am J Gastroenterol 2001 Oct;96(10):2992-3003. [doi: 10.1111/j.1572-0241.2001.04677.x] [Medline: 11693338]

9. Collaborative Group on Hormonal Factors in Breast Cancer. Familial breast cancer: collaborative reanalysis of individual data from 52 epidemiological studies including 58,209 women with breast cancer and 101,986 women without the disease. Lancet 2001 Oct 27;358(9291):1389-1399. [doi: 10.1016/S0140-6736(01)06524-2] [Medline: 11705483]

10. Porta M. A Dictionary of Epidemiology Internet. Oxford, UK: Oxford University Press; 2016. URL: https://www. oxfordreference.com/view/10.1093/acref/9780199976720.001.0001/acref-9780199976720 [accessed 2020-12-07]

11. Pharoah PD, Day NE, Duffy S, Easton DF, Ponder BA. Family history and the risk of breast cancer: a systematic review and meta-analysis. Int J Cancer 1997 May 29;71(5):800-809 [FREE Full text] [doi: 10.1002/(sici)1097-0215(19970529)71:5<800::aid-ijc18>3.0.co;2-b] [Medline: 9180149]

12. Meystre SM, Savova GK, Kipper-Schuler KC, Hurdle JF. Extracting information from textual documents in the electronic health record: a review of recent research. Yearb Med Inform 2008:128-144. [Medline: 18660887]

13. Wang Y, Wang L, Rastegar-Mojarad M, Moon S, Shen F, Afzal N, et al. Clinical information extraction applications: A literature review. J Biomed Inform 2018 Jan;77:34-49 [FREE Full text] [doi: 10.1016/j.jbi.2017.11.011] [Medline: 29162496]

14. Wu S, Roberts K, Datta S, Du J, Ji Z, Si Y, et al. Deep learning in clinical natural language processing: a methodical review. J Am Med Inform Assoc 2020 Mar 01;27(3):457-470. [doi: 10.1093/jamia/ocz200] [Medline: 31794016]

15. Uzuner Ö, South BR, Shen S, DuVall SL. 2010 i2b2/VA challenge on concepts, assertions, and relations in clinical text. J Am Med Inform Assoc 2011;18(5):552-556 [FREE Full text] [doi: 10.1136/amiajnl-2011-000203] [Medline: 21685143]

16. Suominen H, Salanterä S, Velupillai S, Chapman W, Savova G, Elhadad N, et al. Overview of the ShARe/CLEF eHealth Evaluation Lab 2013. In: Forner P, Müller H, Paredes R, Rosso P, Stein B, editors. Information Access Evaluation. Multilinguality, Multimodality, and Visualization. Berlin, Heidelberg: Springer; 2013:212-231.

17. Kelly L, Goeuriot L, Suominen H, Schreck T, Leroy G, Mowery D, et al. Overview of the ShARe/CLEF eHealth Evaluation Lab 2014. In: Information Access Evaluation. Multilinguality, Multimodality, and Interaction. New York City, USA: Springer; 2014:172-191.

18. Henry S, Buchan K, Filannino M, Stubbs A, Uzuner O. 2018 n2c2 shared task on adverse drug events and medication extraction in electronic health records. J Am Med Inform Assoc 2020 Jan 01;27(1):3-12. [doi: 10.1093/jamia/ocz166] [Medline: $\underline{31584655]}$

19. Jagannatha A, Liu F, Liu W, Yu H. Overview of the First Natural Language Processing Challenge for Extracting Medication, Indication, and Adverse Drug Events from Electronic Health Record Notes (MADE 1.0). Drug Saf 2019 Jan;42(1):99-111 [FREE Full text] [doi: 10.1007/s40264-018-0762-z] [Medline: $\underline{\text { 30649735] }}$

20. Sun W, Rumshisky A, Uzuner O. Evaluating temporal relations in clinical text: 2012 i2b2 Challenge. J Am Med Inform Assoc 2013;20(5):806-813 [FREE Full text] [doi: 10.1136/amiajnl-2013-001628] [Medline: 23564629]

21. Aronson AR, Lang F. An overview of MetaMap: historical perspective and recent advances. J Am Med Inform Assoc 2010;17(3):229-236 [FREE Full text] [doi: 10.1136/jamia.2009.002733] [Medline: 20442139]

22. Savova GK, Masanz JJ, Ogren PV, Zheng J, Sohn S, Kipper-Schuler KC, et al. Mayo clinical Text Analysis and Knowledge Extraction System (cTAKES): architecture, component evaluation and applications. J Am Med Inform Assoc 2010;17(5):507-513 [FREE Full text] [doi: 10.1136/jamia.2009.001560] [Medline: 20819853]

23. Soysal E, Wang J, Jiang M, Wu Y, Pakhomov S, Liu H, et al. CLAMP - a toolkit for efficiently building customized clinical natural language processing pipelines. J Am Med Inform Assoc 2018 Mar 01;25(3):331-336. [doi: 10.1093/jamia/ocx132] [Medline: 29186491]

24. Wang A, Singh A, Michael J, Hill F, Levy O, Bowman S. GLUE: A Multi-Task Benchmark and Analysis Platform for Natural Language Understanding. arXiv 2019 [FREE Full text]

25. Lample G, Ballesteros M, Subramanian S, Kawakami K, Dyer C. Neural Architectures for Named Entity Recognition. arXiv 2016 [FREE Full text]

26. Devlin J, Chang M, Lee K, Toutanova K. BERT: Pre-training of Deep Bidirectional Transformers for Language Understanding. arXiv 2018 [FREE Full text]

27. Lee J, Yoon W, Kim S, Kim D, Kim S, So CH, et al. BioBERT: a pre-trained biomedical language representation model for biomedical text mining. Bioinformatics 2020 Feb 15;36(4):1234-1240. [doi: 10.1093/bioinformatics/btz682] [Medline: 31501885]

28. Alsentzer E, Murphy J, Boag W, Weng W, Jindi D, Naumann T, et al. Publicly Available Clinical BERT Embeddings. arXiv 2019 [FREE Full text]

29. Peng Y, Yan S, Lu Z. Transfer Learning in Biomedical Natural Language Processing: An Evaluation of BERT and ELMo on Ten Benchmarking Datasets. arXiv 2019 [FREE Full text]

30. Wang Y, Wang L, Rastegar-Mojarad M, Liu S, Shen F, Liu H. Systematic Analysis of Free-Text Family History in Electronic Health Record. AMIA Jt Summits Transl Sci Proc 2017;2017:104-113 [FREE Full text] [Medline: 28815117]

31. Goryachev S, Kim H, Zeng-Treitler Q. Identification and extraction of family history information from clinical reports. AMIA Annu Symp Proc 2008 Nov 06:247-251 [FREE Full text] [Medline: 18999129]

32. Bill R, Pakhomov S, Chen ES, Winden TJ, Carter EW, Melton GB. Automated extraction of family history information from clinical notes. AMIA Annu Symp Proc 2014;2014:1709-1717 [FREE Full text] [Medline: 25954443] 
33. Liu S, Wang Y, Liu H. Selected articles from the BioCreative/OHNLP challenge 2018. BMC Med Inform Decis Mak 2019 Dec 27;19(Suppl 10):262 [FREE Full text] [doi: 10.1186/s12911-019-0994-6] [Medline: $\underline{31882003}$ ]

34. Sijia L, Majid RM, Yanshan W, Liwei W, Feichen S, Sunyang F, et al. Overview of the BioCreative/OHNLP 2018 Family History Extraction Task. 2018. URL: https://www.researchgate.net/publication/ 327424806 Overview of the BioCreativeOHNLP 2018 Family History Extraction Task [accessed 2020-12-10]

35. Shi X, Jiang D, Huang Y, Wang X, Chen Q, Yan J, et al. Family history information extraction via deep joint learning. BMC Med Inform Decis Mak 2019 Dec 27;19(Suppl 10):277 [FREE Full text] [doi: 10.1186/s12911-019-0995-5] [Medline: 31881967]

36. Brill E. Automatic Grammar Induction and Parsing Free Text: A Transformation-Based Approach. 1993 Presented at: The 31st Annual Meeting of the Association for Computational Linguistics; 1993; Columbus, OH p. 259-265 URL: https://www. aclweb.org/anthology/P93-1035/ [doi: 10.3115/981574.981609]

37. Ramshaw L, Marcus M. Text Chunking using Transformation-Based Learning. arXiv 1995 [FREE Full text]

38. Nayel H, Shashirekha H. Improving NER for Clinical Texts by Ensemble Approach using Segment Representations. 2017 Presented at: The 14th International Conference on Natural Language Processing (ICON-2017); 2017; Kolkata, West Bengal, India p. 197-204 URL: https://www.aclweb.org/anthology/W17-7525

39. Wei Q, Ji Z, Li Z, Du J, Wang J, Xu J, et al. A study of deep learning approaches for medication and adverse drug event extraction from clinical text. J Am Med Inform Assoc 2020 Jan 01;27(1):13-21 [FREE Full text] [doi: 10.1093/jamia/ocz063] [Medline: 31135882$]$

40. Kim Y, Meystre SM. Ensemble method-based extraction of medication and related information from clinical texts. $\mathrm{J}$ Am Med Inform Assoc 2020 Jan 01;27(1):31-38 [FREE Full text] [doi: 10.1093/jamia/ocz100] [Medline: $\underline{31282932}$ ]

41. Christopoulou F, Tran TT, Sahu SK, Miwa M, Ananiadou S. Adverse drug events and medication relation extraction in electronic health records with ensemble deep learning methods. J Am Med Inform Assoc 2020 Jan 01;27(1):39-46 [FREE Full text] [doi: 10.1093/jamia/ocz101] [Medline: 31390003]

42. Dai H. Family member information extraction via neural sequence labeling models with different tag schemes. BMC Med Inform Decis Mak 2019 Dec 27;19(Suppl 10):257 [FREE Full text] [doi: 10.1186/s12911-019-0996-4] [Medline: 31881965]

43. Tang B, Wu Y, Jiang M, Chen Y, Denny JC, Xu H. A hybrid system for temporal information extraction from clinical text. J Am Med Inform Assoc 2013;20(5):828-835 [FREE Full text] [doi: 10.1136/amiajnl-2013-001635] [Medline: 23571849]

44. Yang X, Bian J, Gong Y, Hogan WR, Wu Y. MADEx: A System for Detecting Medications, Adverse Drug Events, and Their Relations from Clinical Notes. Drug Saf 2019 Jan 2;42(1):123-133 [FREE Full text] [doi: 10.1007/s40264-018-0761-0] [Medline: $\underline{30600484]}$

45. Yang X, Bian J, Fang R, Bjarnadottir RI, Hogan WR, Wu Y. Identifying relations of medications with adverse drug events using recurrent convolutional neural networks and gradient boosting. J Am Med Inform Assoc 2020 Jan 01;27(1):65-72 [FREE Full text] [doi: 10.1093/jamia/ocz144] [Medline: 31504605]

46. Yang X, Lyu T, Li Q, Lee C, Bian J, Hogan WR, et al. A study of deep learning methods for de-identification of clinical notes in cross-institute settings. BMC Med Inform Decis Mak 2019 Dec 05;19(Suppl 5):232 [FREE Full text] [doi: 10.1186/s12911-019-0935-4] [Medline: 31801524]

47. Bojanowski P, Grave E, Joulin A, Mikolov T. Enriching Word Vectors with Subword Information. TACL 2017 Dec;5:135-146. [doi: 10.1162/tacl a 00051]

48. Vaswani A, Shazeer N, Parmar N, Uszkoreit J, Jones L, Gomez A, et al. Attention is All you Need. arXiv 2017 [FREE Full text]

49. Wu Y, Yang X, Bian J, Guo Y, Xu H, Hogan W. Combine Factual Medical Knowledge and Distributed Word Representation to Improve Clinical Named Entity Recognition. AMIA Annu Symp Proc 2018;2018:1110-1117 [FREE Full text] [Medline: 30815153]

50. Wolf T, Debut L, Sanh V, Chaumond J, Delangue C, Moi A, et al. HuggingFace's Transformers: State-of-the-art Natural Language Processing. arXiv 2020 [FREE Full text]

51. Paszke A, Gross S, Massa F, Lerer A, Bradbury J, Chanan G, et al. PyTorch: An Imperative Style, High-Performance Deep Learning Library. arXiv 2019 [FREE Full text]

52. Si Y, Wang J, Xu H, Roberts K. Enhancing clinical concept extraction with contextual embeddings. J Am Med Inform Assoc 2019 Nov 01;26(11):1297-1304. [doi: 10.1093/jamia/ocz096] [Medline: 31265066 ]

53. Soares L, FitzGerald N, Ling J, Kwiatkowski T. Matching the Blanks: Distributional Similarity for Relation Learning. arXiv 2019 [FREE Full text]

54. Frangi AF, Tsaftaris SA, Prince JL. Simulation and Synthesis in Medical Imaging. IEEE Trans Med Imaging 2018 Mar;37(3):673-679. [doi: 10.1109/tmi.2018.2800298]

55. Yi X, Walia E, Babyn P. Generative adversarial network in medical imaging: A review. Med Image Anal 2019 Dec;58:101552. [doi: 10.1016/j.media.2019.101552] [Medline: 31521965]

56. Guan J, Li R, Yu S, Zhang X. Generation of Synthetic Electronic Medical Record Text. 2018 Presented at: IEEE International Conference on Bioinformatics and Biomedicine (BIBM); 2018; Madrid, Spain p. 374-380. [doi: 10.1109/BIBM.2018.8621223] 
57. Yang X, He X, Zhang H, Bian J, Wu Y. UF team in 2019 N2C2 challenge Track2 Family history extraction from clinical narratives. 2019. URL: https://github.com/uf-hobi-informatics-lab/2019 N2C2 Track2 FHextraction.git [accessed 2020-12-07]

\author{
Abbreviations \\ BERT: bidirectional encoder representations from transformers \\ BIO: beginning-inside-outside \\ CRFs: conditional random fields \\ FH: family history \\ LSTM: long short-term memory \\ n2c2: National NLP Clinical Challenge \\ NER: named entity recognition \\ NLP: natural language processing \\ GPU: graphics processing unit
}

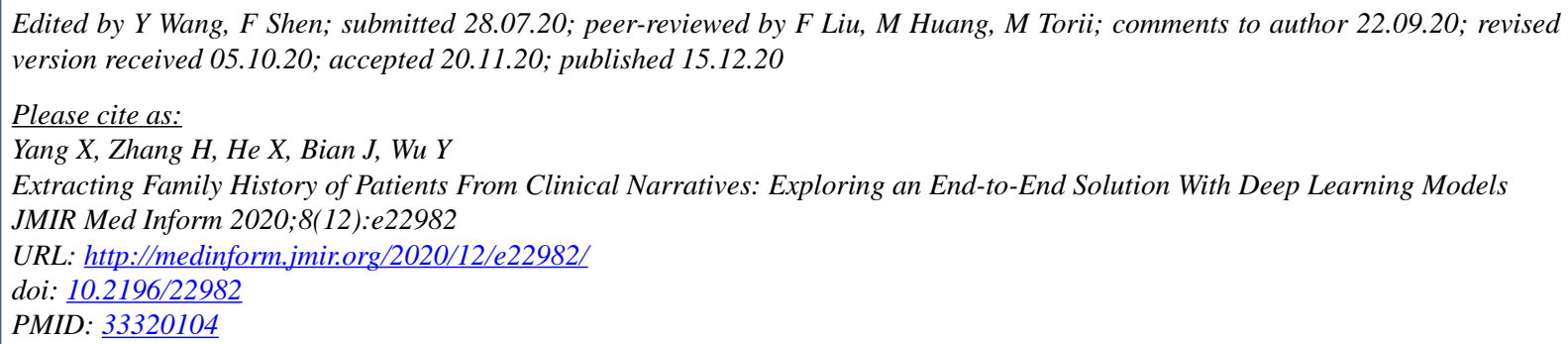

(CXi Yang, Hansi Zhang, Xing He, Jiang Bian, Yonghui Wu. Originally published in JMIR Medical Informatics (http://medinform.jmir.org), 15.12.2020. This is an open-access article distributed under the terms of the Creative Commons Attribution License (https://creativecommons.org/licenses/by/4.0/), which permits unrestricted use, distribution, and reproduction in any medium, provided the original work, first published in JMIR Medical Informatics, is properly cited. The complete bibliographic information, a link to the original publication on http://medinform.jmir.org/, as well as this copyright and license information must be included. 